\title{
Light Scattering of High Polymer Solutions*
}

\author{
JURg Waser, Richard M. Badger, ANd Verner Schomaker \\ The Gates and Crellin Laboratories of Chemistry, California Institute of Technology, Pasadena, California
}

(Received November 24, 1945)

$I^{\mathrm{N}}$ $\mathrm{N}$ principle it is possible to determine the molecular weight distribution of a high polymer, containing no excessively large molecules, from interpretation of its light scattering in solutions at various concentrations. Actually there appear to be severe limitations to this method. Not only is it evident that, considering experimental error, the resolving power is low for the detection in any part of the molecular weight distribution curve of a group of components of similar molecular weights (as contrasted to a single component), it may also happen, in consequence of the restriction of the data to a certain concentration range, that components at the ends of the distribution curve will not be detected and, moreover, that the conclusions regarding the remainder of the distribution will be distorted.

In particular it may be difficult or impossible to perform a satisfactory extrapolation to infinite dilution. In a recent letter to the Editor of this Journal $^{1}$ and in a later article ${ }^{2}$ Zimm, Doty, and Mark have used the expansion formula

$$
\begin{gathered}
H \frac{c}{\tau_{2}}=\frac{1}{\sum_{i} \frac{1}{\frac{f_{i}}{M_{i}}+\frac{2 B}{R T} c+\frac{3 C}{R T} c^{2}+\cdots}=\frac{1}{\bar{M}}+\frac{2 B}{R T}\left[1+\left\langle\left(\frac{\Delta M}{\bar{M}}\right)^{2}\right\rangle_{\mathrm{AV}}\right] c} \\
+\left\{\frac{3 C}{R T}\left[1+\left\langle\left(\frac{\Delta M}{\bar{M}}\right)^{2}\right\rangle_{\mathrm{Av}}\right]_{t}+\frac{4 B^{2} \bar{M}}{R^{2} T^{2}}\left[\left(\left\langle\left(\frac{\Delta M}{\bar{M}}\right)^{2}\right\rangle_{\mathrm{Av}}\right)^{2}-\left\langle\left(\frac{\Delta M}{\bar{M}}\right)^{2}\right\rangle_{\mathrm{AV}}-\left\langle\left(\frac{\Delta M}{\bar{M}}\right)^{3}\right\rangle_{\mathrm{Av}}\right\} c^{2}+\cdots\right.
\end{gathered}
$$

Here $H$ is a constant, $\tau_{2}$ the turbidity due to the solute, $c$ the concentration of the polymer in grams per milliliter of solution, $f_{i}$ the weight fraction in the polymer of the species of molecular weight $M_{i}$, and $B$ and $C$ are constants occurring in the expression for the osmotic pressure (see below). The weight average molecular weight is denoted by $\bar{M}$, and $\left\langle(\Delta M / \bar{M})^{2}\right\rangle_{\mathrm{Av}}$ and $\left\langle(\Delta M / \bar{M})^{3}\right\rangle_{\mathrm{Av}}$ are the second and third moments of the molecular weight distribution curve about the weight average molecular weight. Expansion (1) may however fail even at the low concentrations used by the above authors. For example in their letter ${ }^{1}$ experimental points are plotted for the light scattering of a mixture of two homogeneous polystyrene fractions and compared with the parabola corresponding to the right-hand side of (1). If, however, the complete expression on the left-hand side of (1) is plotted for the mixture of the two fractions, the resulting curve is found

* Contribution No. 1024 from the Gates and Crellin Laboratories of Chemistry. to deviate markedly from the parabola at concentrations approaching one percerit, and to be much less curved. It is therefore evident that expansion (1) may be misleading even at reasonably low concentrations.

It is to be emphasized that expansion (1) may either be satisfactory at moderate concentrations, somewhat misleading as in Zimm, Doty, and Mark's example, or wholly inadequate, depending upon the nature of the molecular weight distribution and osmotic behavior (e.g., the values of $B, C, \cdots$ in Eq. (1)) of the polymer. We now give some rather general considerations which possibly add something to our understanding of this situation.

The equations which must be handled are considerably simplified and the considerations in general are facilitated by the use of P. Debye's

${ }^{1}$ B. H. Zimm and P. M. Doty, J. Chem. Phys. 12, 203 (1944).

2 P. M. Doty, B. H. Zimm, and H. Mark, J. Chem. Phys. 13,159 (1945). 


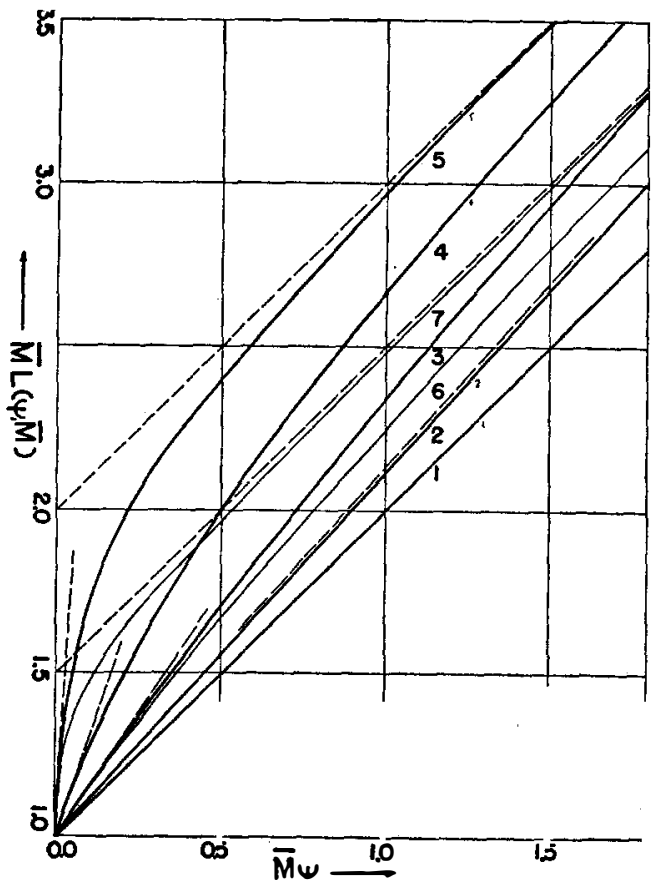

FIG. 1. Plot of $L(\psi)$ for various two-component distributions. Curve 1 represents a monodisperse system, curves 2-5 (heavily drawn) tend toward the upper asymptote, and curves 6 and 7 (lightly drawn) belong to the lower asymptote. The limiting slopes are also indicated. The parameters involved are the following:

\begin{tabular}{|c|c|c|c|c|c|}
\hline & $\frac{\bar{M}}{\bar{M}_{n}}$ & & $\frac{M_{1}}{\overline{\bar{M}}}$ & $\frac{M_{2}}{\bar{M}}$ & $f_{1}$ \\
\hline $\begin{array}{l}(1) \\
(2) \\
(3) \\
(4) \\
(5) \\
(6) \\
(7)\end{array}$ & $\begin{array}{l}1 \\
2 \\
2 \\
2 \\
2 \\
1.5 \\
1.5\end{array}$ & $\begin{array}{r}0 \\
\frac{1}{8} \\
\frac{1}{2} \\
2 \\
16 \\
\frac{1}{2} \\
16\end{array}$ & $\begin{array}{c}1 \\
1.14 \\
1.71 \\
42.56 \\
32.5 \\
2.0 \\
48.3\end{array}$ & $\begin{array}{l}0 \\
0.11 \\
.29 \\
.44 \\
.50 \\
.50 \\
.66\end{array}$ & $\begin{array}{l}1 \\
0.86 \\
.50 \\
.14 \\
.016 \\
.333 \\
.007\end{array}$ \\
\hline
\end{tabular}

suggestion $^{3}$ that $H c / \tau_{2}$ be considered as function of a quantity $\psi(c)$,

$$
H \underset{\tau_{2}}{\frac{c}{\sum_{i}}}=\frac{1}{\frac{f_{i} M_{i}}{1+\psi M_{i}}}=L(\psi),
$$

where $\psi$ is defined as follows. Let the osmotic pressure be given the representation

$$
\pi=R T\left\{\sum_{i} \frac{f_{i}}{M_{i}} c+\varphi(c)\right\},
$$

in which $\varphi(c)$, whose first derivative vanishes at zero concentration, is independent of the

${ }^{3}$ P. Debye, General Report No. 17, August 28, 1944. War Production Board, Office of Assistant Rubber Director of Research and Development of Synthetics. degree of polymerization; ${ }^{4}$ then $\psi$ is given by $\psi(c)=(d / d c) \varphi(c) . \quad(\operatorname{In} \quad(1) \quad \psi=(2 B / R T) c$ $+(3 C / R T) c^{2}+\cdots$ was used.) The form of $\psi(c)$ can be determined either by osmotic pressure studies or by measurements of the light scattering of monodisperse fractions.

The following general remarks can be made about $L(\psi)$ :

(a) The function (3) has always a positive slope

$$
\frac{\partial L}{\partial \psi}=L^{2} \sum_{i} \frac{f_{i} M_{i}^{2}}{\left(1+\psi M_{i}\right)^{2}}
$$

and is never curved upward, since

$\frac{\partial^{2} L}{\partial \psi^{2}}=-L^{3} \sum_{i j} \frac{f_{i} M_{i} f_{j} M_{j}}{\left(1+\psi M_{i}\right)^{3}\left(1+\psi M_{j}\right)^{3}}\left(M_{i}-M_{j}\right)^{2} \leq 0$.

It is evident that in general $\partial^{2} L / \partial \psi^{2}$, and hence the deviation of $L(\psi)$ from a straight line, is greater, the greater the heterogeneity of the mixture.

(b) For a polydisperse system $L(\psi)$ is a curve whose initial slope is $\left(1+\left\langle(\Delta M / \bar{M})^{2}\right\rangle_{\mathrm{Av}}\right)^{1,2}$ and whose intercept is the weight average molecular weight. For the theoretical limit of large $\psi$, such that $\psi M_{i} \gg 1, L(\psi)$ approaches an asymptote of unit slope, whose intercept is the reciprocal number average molecular weight $1 / \bar{M}_{n}$. According to this and Eq. (5) the slope of $L(\psi)$ is always greater than unity.

(c) For a monodisperse system $L(\psi)$ is simply a straight line of unit slope whose intercept is the reciprocal of the molecular weight.

To show some of the wide possible variations in the form of $L(\psi)$ we present theoretical plots for mixtures with various values of $\bar{M} / \bar{M}_{n}$ and involving solute molecules of only two different degrees of polymerization. The graphs are in terms of $\bar{M} L\left(\psi, M_{i}\right), M_{i} / \bar{M}$ and $\psi \bar{M}$, since $\bar{M} L\left(\psi, M_{i}\right)=L\left(\bar{M} \psi, M_{i} / \bar{M}\right)$. (Figure 1.)

It should be noted that for any solution obeying Eq. (2) (and the curves given here for bimodal distributions illustrate this) any measurement of $L$ provides an upper limit for $\bar{M}_{n}$ and a lower

${ }^{4}$ For experimental evidence that this is a good approximation cf. e.g., M. L. Huggins, J. Am. Chem. Soc. 64, 1712 (1942); T. Alfrey, A. Bartovics, and H. Mark, J. Am. Chem. Soc. 65, 2319 (1943). 
limit for $\bar{M}$, while any two measurements of $L$ at different $\psi$ establish a lower limit for $\left\langle(\Delta M / \bar{M})^{2}\right\rangle_{\mathrm{AV}}$. Whether these limits lie close to the actual values of the corresponding quantities depends on the curves and the values of $\bar{M} \psi$ in question: for any range of $\bar{M} \psi$ with $\left\langle(\Delta M / \bar{M})^{2}\right\rangle_{A v}$ small enough compared to $\bar{M} / \bar{M}_{n}$ satisfactory extrapolation to $\psi=0$ (as with the aid of Eq. (1)) is feasible for determining $\left\langle(\Delta M / \bar{M})^{2}\right\rangle_{A v}$ and $\bar{M}$, and with $\left\langle(\Delta M / \bar{M})^{2}\right\rangle_{\mathrm{Av}} /\left(\bar{M} / \bar{M}_{n}\right)$ great enough the estimate of $1 / \bar{M}_{n}$ will be satisfactory, but generally the range of $\psi$ in which significant measurements can be made may not be wide enough either to satisfy both these conditions or to make certain whether one of them is really satisfied. If extrapolation to $\psi=0$ appears to be possible it may still be unsatisfactory because of the presence of a relatively small amount of material of relatively extreme high molecular weight such that the correct curve of $L(\psi)$ bends significantly downward at values of $\psi$ lower than that of the lowest measurement. On the other hand, in the presence of material of relatively extreme low molecular weight, the curve of $L(\psi)$ may have a maximum slope very little greater than unity and may seem to have reached an asymptote at the highest attained value of $\psi$ when actually its asymptote lies higher. 